\title{
128. Anomalies optiques présentées par les calcites soumises à l'action des compressions.
}

\author{
Par Shigeru Nishro.
}

(Regu le 15 sept. Connu K. TAwARA, M.I.A., le 12 oct. 1926)

Des expériences précises ont déjà été tentées sur la question de la compression des roches, mais les transformations subies par les minéraux constitutifs des roches comprimées n'ont pu être encore clairement définis.

Afin de mieux suivre les diverses phases de transformation qui se produisent au cours des expériences, des études optiques ont été entreprises sur les calcaires cristallins avant et après compression. Les pièces d'essai sur lesquelles se font les expériences sont empruntées aux calcaires de Hinami (province d'Okayama) qui se rattachent à la période paléozoïque, et à ceux de Hitachi (prorince d'Tbaraki) dans lesquels prédominent les terrains précambriens.

Ces pièces d'essai, préalablement taillées en forme cubique, sont sonmises à l'action de la presse hydraulique. Les contractions se font perpendiculairement. On enregistre d'une part les nourelles charges ajoutées à la presse, et de l'autre les diverses contractions éprouvées jar les pièces d'essai; puis l'on détermine le point où la roche commence à se désagréger. Pour savoir maintenent comment se comportent les roches comprimées entre des surfaces résistantes, comme cela se passe à l'intérieur des couches de la croûte terrestre, les pièces d'essai sont encastrées dans un cadre rigide en acier fondu, et la compression se fait de nouveau verticalement. On constate que, dans ce dernier cas, Ia roche résiste mieux à l'action de la compression et que son point de désagrégation n'est atteint qu'au prix d'une charge double.

En second lieu, les matériaux d'essai sont taillés en forme de lames minces et leurs transformations sont observées à l'aide du microscope polarisant. Les calcaires de Hinami employés dans l'expérimentation sont composés de grains de calcite disposés en mosaïque; les clivages suivent les directions des faces d'un rhomboèdre, les macles rhomboèdres sont parfaites, et sous l'action de la lumière convergente apparaît la figure intervenue caractéristique d'un cristal uniaxe. Après compression, on remarque que les grains cristallins se sont déformés et ont rerêtu une 
contexture schisteuse; les stries de clivage se sont incurvées, des macles nouvelles se sont produites par voie de glissement, et la figure intervenue donne l'aspect de deux axes optiques qui sont uniquement négatifs. Les angles des axes optiques sont déterminées par la méthode de Fedorow en faisant usage de table rotatoire universale. Elles varient par des grains de calcite; une des elles est d'environ $13^{\prime}$ pour la ligne $\mathbf{D}$. Nous avons donc remarqué que les calcites, dans les calcaires comprimés, donnent naissance à des anomalies optiques.

Telles sont les modifications éprouvées par les calcites dans la compression artificielle. Dans la nature cependant on rencontre parfois un pareil métamorphisme. Prenons par exemple un échantillon de roche de Hitachi comprimée naturellement et étudions-le sous forme de lames minces. Nous observons non seulement une structure cristallophyllienne de calcite, mais encore, comme dans le cas de compression artificielle, des phénomènes optiques anomaux qui semblent bien être le résultat d'une pression latérale éprouvée au cours de grandes transformations de la croûte terrestre. C'est ainsi que l'on peut observer sur place, des calcaires, dont la direction en longueur nord nord-est, sont localisés dans les régions où se sont fait sentir des plissements énergiques. On reconnait donc nettement les compressions latérales s'exerçant dans les,directions ouest ouest-nord ou est est-sud.

Comme la calcite est originairement un cristal uniaxe négatif, on peut considérer comme spécial le cas où l'angle des axes optiques d'un cristal biaxe négatif est zéro. Aussi, si l'on admet qu'elle a subi une déviation mécanique dans sa structure interne, il est facile de conclure qu'elle se transforme, avant tout, au biaxe négatif.

Afin de préciser les rapports mutuels qui existent entre les axes optiques et la symétrie dans les phénomènes de dispersion des axes optiques, nous avons pris les évaluations en faisant usage de rayons monochromatiques visibles. Si l'on fait varier la valeur $430 \mu \mu$ de la la longueur d'onde jusqu'à $656 \mu \mu$, on peut alors constater un changement régulier dans les angles optiques, dont la bissectrice se déplace dans le même plan, c'est-à-dire qu'il se produit la dispersion inclinée de Des Cloizeaux, indiquée par la notation $\rho>v$. Le phénomène présenté par les calcaires de Hinami pressés artificiellement est tout à fait identique à celui des calcaires de Hitachi ayant subi un dynamométamorphisme.

A la fin de l'expérience, nous avons pris des photographies aux rayons $X$. Celles des calcaires de Hinami présentent l'aspect de tache de Laue dont les multiples combinaisons de tache sont produites par la 
réflexion des rayons $\mathrm{X}$ sur les cristaux de calcite orientés dans diverses directions. Quant au calcaire comprimé artificiellement ou métamorphosé à l'intérieur des couches terrestres présente un aspect tout différent. En effet la photographie nous donne une figure rayonnante comparable à celle que présentent les corps fibreux. Les résultats observés à l'aide des rayons $\mathrm{X}$ sont done les similaires à ceux que présente l'observation microscopique.

Nous croyons avoir donné une idée suffisante des caractères essentiels des anomalies optiques. Au point de vue géologique, le calcaire est une des roches les plus répandues non seulement géographiquement mais encore à travers une longue période géologique. En résumé, on peut conclure que toute pression s'exerçant sur les calcaires, quelle qu'en soit l'origine, produit nécessairement des anomalies optiques. On peut, d'autre part, par la simple observation de leurs caractères optiques, deviner la nature des calcaires, lorsque leur métamorphisme s'est opéré dans les profondeurs moindres de la croûte terrestre.

\author{
Institut de Mine, \\ Université Impériale de Tokyo.
}

